\title{
DESIGN OF THIN COMPOSITE SKINS OF ANISOTROPIC STRUCTURE OF BEARING PANELS OF AIRCRAFT STRUCTURES IN POST-BUCKLING STATE UNDER COMBINED LOADING
}

\author{
Oleg Mitrofanov ${ }^{1}$, Igors Lebedevs ${ }^{2}$, Margarita Urbaha ${ }^{2}$ \\ ${ }^{1}$ Moscow Aviation Institute (National Research University), Russia; \\ ${ }^{2}$ Riga Technical University, Latvia \\ mitrofanovov@mai.ru,igors.lebedevs@aviatest.lv,margarita.urbaha@rtu.lv
}

\begin{abstract}
Applied investigations of composite aircraft structures show that thin walls or panels of an anisotropic structure are effective in some cases. For thin-walled structures at loads above the operational level, buckling is allowed. Moreover, the task of design based on the post-buckling state becomes much more complicated when it is necessary to take into account the combined loading under biaxial compression and shear. The supercritical behaviour of anisotropic structure's thin panels geometrically nonlinear relations is presented in this paper. For satisfactory description of the panel's supercritical behaviour under hinged support combined loading the form of the deflection function is proposed. The analytical solution based on the Bubnov-Galerkin method how to determine the nonlinear stress-strain state allows obtaining a nonlinear equation connecting the acting flows with the deflection amplitude. Based on the definition of the stress function, equations are written for calculating all components of membrane stresses. Also, expressions are presented for determining bending stresses. It is desirable to take into account when the stress-strain state for geometrically nonlinear behaviour of panels of medium thickness has been determined. The relations of the mechanics for composite panels are presented and, also, the general form of equality for the stresses acting in a monolayer of the composite under combined loading. Further, when using Tsai's criterion for a monolayer, as well for the received nonlinear equations of the stress-strain state with supercritical behaviour, a nonlinear equation was obtained to determine the optimal thickness of the anisotropic panel under its combined loading.
\end{abstract}

Keywords: post-buckling state, anisotropic structure, rectangular panel, compression, shear.

\section{Introduction}

When designing thin panels and walls of structures of wing mechanization units for aircraft of low and medium carrying capacity under loading above the operational level, loss of stability is allowed [1]. In certain situations, when considering multiple loading cases, anisotropic panels and walls can be effective. The nature of loading in the general case can be of a combined nature under biaxial compression and shear. Moreover, taking into account all loading components is important in subsequent calculations of resource characteristics.

The development of composite structures presupposes the search for solutions to many complex urgent problems. Note that the analysis of the combined loading of composite panels is devoted to the works [2-3]. Monographs [4-6] include the most significant modern theories of calculation and design of composite thin-walled structures. Also interesting are the works [7-8] devoted to analytical studies of the supercritical behaviour of composite panels.

Next, we will use the methodology for designing composite panels, which is based on the use of analytical solutions of geometrically nonlinear problems and the achievement of ultimate strength stresses during panel buckling [9-10]. For example, consider the design ratios for determining the thicknesses of orthotropic rectangular panels under uniaxial compression [10]. From the conditions for ensuring strength and stability, we have (Table 1) known relations with linear and quadratic equations. In the case of supercritical design, an analytical solution to a geometrically nonlinear problem was required. In Table 1, for this case, an equation is given for determining the membrane longitudinal stresses, as well as equality, which is the result of solving a nonlinear problem by the Bubnov Galerkin method. When considering the two indicated equations, provided that the ultimate in strength normal stresses are achieved, the design problem for the supercritical state is reduced to a cubic equation with respect to the desired thickness of the composite panel.

The purpose of this work was to develop an applied technique for determining the minimum thickness of an anisotropic rectangular panel with hinged support under conditions of combined loading by compressive and tangential flows with supercritical behaviour. The technique should be based on an analytical solution of a geometrically nonlinear problem, and the obtained relationships should allow at 
the early design stages to carry out expert assessments of thicknesses, taking into account the supercritical behaviour of the panels.

Design ratios for determining the thickness of an orthotropic rectangular panel under uniaxial compression [10]

\begin{tabular}{|c|c|c|}
\hline $\begin{array}{c}\text { Panel esigning } \\
\text { terms }\end{array}$ & Stress computation formula & $\begin{array}{c}\text { Determination of panel minimal } \\
\text { thickness }\end{array}$ \\
\hline Static Strength & $\sigma=\frac{P}{\delta b}$ & $\delta=\frac{P}{\bar{\sigma} b}$ \\
\hline Stability & $\sigma_{\kappa p}=K\left(\frac{\delta}{b}\right)^{2}, K=\frac{2 \pi^{2}}{12} \times$ & $\delta^{2}=b^{2} \frac{\bar{\sigma}}{K}$ \\
\hline$\times\left[\sqrt{\overline{\mathrm{E}}_{\mathrm{x}} \overline{\mathrm{E}}_{\mathrm{y}}}+\mu_{\mathrm{xy}} \overline{\mathrm{E}}_{\mathrm{x}}+2 \mathrm{G}_{\mathrm{xy}}\right\rfloor$ & $\sigma_{x}=-\frac{f^{2}}{8} E_{x} \frac{\pi^{2} m^{2}}{a^{2}}-p_{x}$, \\
$\begin{array}{c}\text { Post-buckling } \\
\text { state }\end{array}$ & $\delta^{2} \bar{D}_{m n}+f^{2} E_{m n}=p_{x}\left(\frac{m}{a}\right)^{2}$ & $\delta^{3}+\delta \frac{E_{m n} \bar{\sigma}_{x}}{\bar{D}_{m n} E_{\alpha \beta}}-\frac{q_{x}}{\bar{D}_{m n}}\left(\frac{m^{2}}{a^{2}}+\frac{E_{m n}}{E_{\alpha \beta}}\right)=0$ \\
\hline
\end{tabular}

Note: $K, D_{m n}, E_{m n}, E_{\alpha \beta}$ - factors dependent of stiffness properties of panel's composite structure [10], $\bar{\sigma}-$ safe stresses, $q_{x}=p_{x} \delta$ - compression flow on a panel

The strength criterion used in the technique should take into account all stresses acting in a monolayer of a composite package. For the design of thin-walled aircraft structures made of carbon fiber reinforced plastics, it is advisable to use, for example, the Tsai criterion in the proposed method. The development of composite structures presupposes the search for solutions to many complex urgent problems. Note that the analysis of the combined loading of composite panels is devoted in the works [2-3]. Monographs [4-6] include the most significant modern theories of calculation and design of composite thin-walled structures. Also interesting are the works [7-8] devoted to analytical studies of the supercritical behavior of composite panels.

\section{Set of problem and basic formula}

Consider an anisotropic rectangular panel loaded with biaxial compression and shear. Let us assume that all of these powers vary in proportion to one parameter. Provided that the supercritical behavior of the panel is acceptable, it is necessary to use geometrically nonlinear relationships. It is also important to choose a "convenient" type of deflection, which would make it possible to obtain an analytical solution to the problem at a given loading. In [8], in the study of stability under a combined load, the following form of deflection was used

$$
W=f \cdot \sin \frac{\pi y}{b} \sin \frac{\pi(x-\alpha y)}{s},
$$

where $\quad \alpha$-tangent of the angle of inclination of the waves during buckling;

$s$ - distance between the nodal lines.

Note that the same type of deflection has been used in the analytical solution of the problem of the supercritical behavior of a composite panel during shear [10]. In this case, let us use equality (1) to solve the problem of the supercritical behaviour of the panel under combined loading. Let us write the first geometrically nonlinear equation of compatibility of deformations of an anisotropic panel in the form $[4 ; 6]$

$$
L_{1}(F)-L_{2}(W)=0,
$$

where

$$
L_{l}(F)=\frac{1}{E_{y}} \frac{\partial^{4} F}{\partial x^{4}}-g_{31} \frac{\partial^{4} F}{\partial x^{3} \partial y}+g_{22} \frac{\partial^{4} F}{\partial x^{2} \partial y^{2}}-g_{13} \frac{\partial^{4} F}{\partial y^{3} \partial x}+\frac{1}{E_{x}} \frac{\partial^{4} F}{\partial y^{4}}
$$




$$
L_{2}(W)=\left(\frac{\partial^{2} W}{\partial x \partial y}\right)^{2}-\frac{\partial^{2} W}{\partial x^{2}} \frac{\partial^{2} W}{\partial y^{2}} ; g_{31}=\frac{\eta_{y, x y}+\eta_{x y, y}}{G_{x y}} ; g_{22}=\frac{1}{G_{x y}}-\frac{\mu_{x y}}{E_{y}}-\frac{\mu_{y x}}{E_{x}} ; g_{13}=\frac{\eta_{x, x y}+\eta_{x y, x}}{G_{x y}} .
$$

The second geometrically nonlinear equation of the Karman mode can be represented as

$$
L_{4}(W)-L_{3}(F, W)=0 \text {, }
$$

where

$$
\begin{gathered}
L_{3}(F, W)=\frac{\partial^{2} F}{\partial y^{2}} \frac{\partial^{2} W}{\partial x^{2}}+\frac{\partial^{2} F}{\partial x^{2}} \frac{\partial^{2} W}{\partial y^{2}}-2 \frac{\partial^{2} F}{\partial x \partial y} \frac{\partial^{2} W}{\partial x \partial y} . \\
L_{4}(W)=\frac{1}{\delta}\left[D_{11} \frac{\partial^{4} W}{\partial x^{4}}+2\left(D_{12}+2 D_{33}\right) \frac{\partial^{4} W}{\partial x^{2} \partial y^{2}}+D_{22} \frac{\partial^{4} W}{\partial y^{4}}+4 D_{16} \frac{\partial^{4} W}{\partial x^{3} \partial y}+4 D_{26} \frac{\partial^{4} W}{\partial x \partial y^{3}}\right]
\end{gathered}
$$

To obtain an analytical solution to the problem of supercritical behaviour let us use the BubnovGalerkin method. After substituting the deflection (1) into expression (2), an equation can be obtained, the solution of which will be a stress function of the type

$$
F=\frac{f^{2}}{32}\left\{\frac{1}{G_{\alpha}}\left(\frac{s}{b}\right)^{2} \cos \frac{2 \pi(x-\alpha y)}{s}+\left(\frac{b}{s}\right)^{2} E_{x} \cos \frac{2 \pi y}{b}\right\}-\frac{p_{x} y^{2}}{2}-\frac{p_{y} x^{2}}{2}+p_{x y} x y,
$$

where

$$
G_{\alpha}=\frac{1}{E_{y}}+g_{31} \alpha+g_{22} \alpha^{2}+g_{13} \alpha^{3}+\frac{\alpha^{4}}{E_{x}}
$$

$p_{x}, p_{y}, p_{x y}\left(q_{x}, q_{y}, q_{x y}\right)$ - stress (flows), forcing on composite panel. equation

The stresses in the middle surface of an unstable orthotropic panel are determined by the following $\sigma_{x}=\frac{\partial^{2} F}{\partial y^{2}}=-f^{2} \Omega_{1}-p_{x}, \sigma_{y}=\frac{\partial^{2} F}{\partial x^{2}}=-f^{2} \Omega_{2}-p_{y}, \tau_{x y}=-\frac{\partial^{2} F}{\partial x \partial y}=f^{2} \Omega_{3}-p_{x y}$,

where

$$
\begin{aligned}
& \Omega_{1}=\frac{\pi^{2}}{8}\left\{\frac{\alpha^{2}}{G_{\alpha} b^{2}} \cos \frac{2 \pi(x-\alpha y)}{s}+\frac{E_{x}}{s^{2}} \cos \frac{2 \pi y}{b}\right\} \\
& \Omega_{2}=\frac{\pi^{2}}{8} \frac{1}{G_{\alpha} b^{2}} \cos \frac{2 \pi(x-\alpha y)}{s} ; \\
& \Omega_{3}=\frac{\pi^{2}}{8} \frac{\alpha}{G_{\alpha} b^{2}} \cos \frac{2 \pi(x-\alpha y)}{s} .
\end{aligned}
$$

Taking into account the geometric nonlinearity from equality (3) and applying the procedure of the Bubnov - Galerkin method we obtain for $f \neq 0$ the following equation

$$
\begin{gathered}
\frac{\pi^{2}}{s^{2}}\left[\frac{\pi^{2} f^{2}}{16 G_{\alpha}}\left(\frac{\alpha}{b}\right)^{2}+\frac{E_{x}}{16} \frac{\pi^{2} f^{2}}{s^{2}}+p_{x}\right]+\left[\frac{\pi^{2} f^{2}}{16 G_{\alpha}}+p_{y}\right]\left(\frac{\pi^{2}}{b^{2}}+\frac{\alpha^{2} \pi^{2}}{s^{2}}\right)+ \\
+\frac{\alpha \pi^{2}}{s^{2}}\left(\frac{\pi^{2} f^{2}}{8 b^{2} G_{\alpha}}+2 p_{x y}\right)-D_{11} \frac{\pi^{4}}{\delta s^{4}}-2 D_{3}\left(\frac{\pi^{2}}{b^{2}}+\frac{\alpha^{2} \pi^{2}}{s^{2}}\right) \frac{\pi^{2}}{\delta s^{2}}- \\
-\frac{D_{22}}{\delta}\left[\left(\frac{\pi^{2}}{b^{2}}+\frac{\alpha^{2} \pi^{2}}{s^{2}}\right)^{2}+\frac{4 \pi^{4} \alpha^{2}}{b^{2} s^{2}}\right]+\frac{4 D_{16}}{\delta} \frac{\alpha \pi^{4}}{s^{4}}+\frac{4 D_{26}}{\delta} \frac{\alpha \pi^{2}}{s^{2}}\left(3 \frac{\pi^{2}}{b^{2}}+\frac{\alpha^{2} \pi^{2}}{s^{2}}\right)=0 .
\end{gathered}
$$

Under the action of flow combination at small deflections expression (6) can be rewritten as 


$$
\begin{gathered}
q_{x}+q_{y}\left(\frac{s^{2}}{b^{2}}+\alpha^{2}\right)+2 \alpha q_{x y}= \\
=\bar{D}_{11} \delta^{3} \frac{\pi^{2}}{s^{2}}+2 \bar{D}_{3} \delta^{3}\left(\frac{\pi^{2}}{b^{2}}+\frac{\alpha^{2} \pi^{2}}{s^{2}}\right)+\bar{D}_{22} \delta^{3}\left[\frac{s^{2}}{\pi^{2}}\left(\frac{\pi^{2}}{b^{2}}+\frac{\alpha^{2} \pi^{2}}{s^{2}}\right)^{2}+\frac{4 \pi^{2} \alpha^{2}}{b^{2}}\right]- \\
-4 \bar{D}_{16} \delta^{3} \frac{\alpha \pi^{4}}{s^{4}}-4 \bar{D}_{26} \delta^{3} \frac{\alpha \pi^{2}}{s^{2}}\left(3 \frac{\pi^{2}}{b^{2}}+\frac{\alpha^{2} \pi^{2}}{s^{2}}\right)
\end{gathered}
$$

where $D_{m n}=D_{m n} \delta^{3}$.

In the general case, the critical parameters of wave formation during loss of stability are determined numerically from equality (7), taking into account the system of equations

$$
\partial q_{x y} / \partial \alpha=0, \partial q_{x y} / \partial s=0 \text {. }
$$

Further, to solve the problem of optimal design to satisfy the safe-life time requirements, a strength criterion is required, that includes all components of the acting stresses. Let us use Tsai's criterion, which is written in the mode [11]

$$
\left(\frac{\sigma_{1}^{(i)}}{\bar{\sigma}_{1}^{(i)}}\right)^{2}-\frac{\sigma_{1}^{(i)} \sigma_{2}^{(i)}}{\bar{\sigma}_{1}^{(i)} \sigma_{2}^{(i)}}+\left(\frac{\sigma_{2}^{(i)}}{\bar{\sigma}_{2}^{(i)}}\right)^{2}+\left(\frac{\tau_{12}^{(i)}}{\bar{\tau}_{12}}\right)^{2}=1,
$$

where $\sigma_{1}{ }^{(i)}\left(\sigma_{2}{ }^{(i)}\right.$ and $\left.\tau_{12}{ }^{(i)}\right)$ - longitudinal (transverse and shear) stresses, acting along the fibers of the monolayer;

$\bar{\sigma}_{I}{ }^{(i)}\left(\bar{\sigma}_{12}{ }^{(i)}\right.$ and $\left.\bar{\tau}_{12}{ }^{(i)}\right)$ - limiting lateral (transverse and shear) stresses in the monolayer.

Composite panel mechanics gives

$$
\begin{aligned}
& \sigma_{1}^{(i)}=\sigma_{x} a_{x}^{(i)}+\sigma_{y} a_{y}^{(i)}+\tau_{x y} a_{x y}^{(i)} ; \\
& \sigma_{2}^{(i)}=\sigma_{x} b_{x}^{(i)}+\sigma_{y} b_{y}^{(i)}+\tau_{x y} b_{x y}^{(i)} ; \\
& \tau_{12}^{(i)}=\sigma_{x} c_{x}^{(i)}+\sigma_{y} c_{y}^{(i)}+\tau_{x y} c_{x y}^{(i)},
\end{aligned}
$$

where

$$
\begin{aligned}
a_{x}^{(i)}=\frac{1}{E_{x}}\left[\left(A_{11}^{i} \cos ^{2} \varphi_{i}+\right.\right. & \left.\left.A_{21}^{i} \sin ^{2} \varphi_{i}+A_{31}^{i} \sin 2 \varphi_{i}\right)-\mu_{y x}\left(A_{12}^{i} \cos ^{2} \varphi_{i}+A_{22}^{i} \sin ^{2} \varphi_{i}+A_{32}^{i} \sin 2 \varphi_{i}\right)\right]+ \\
& +\frac{\eta_{x y, x}}{G_{x y}}\left[A_{13}^{i} \cos ^{2} \varphi_{i}+A_{23}^{i} \sin ^{2} \varphi_{i}+A_{33}^{i} \sin 2 \varphi_{i}\right]
\end{aligned}
$$$$
a_{y}^{(i)}=\frac{1}{E_{y}}\left[-\mu_{x y}\left(A_{11}^{i} \cos ^{2} \varphi_{i}+A_{21}^{i} \sin ^{2} \varphi_{i}+A_{31}^{i} \sin 2 \varphi_{i}\right)+\left(A_{12}^{i} \sin ^{2} \varphi_{i}+A_{22}^{i} \cos ^{2} \varphi_{i}+A_{32}^{i} \sin 2 \varphi_{i}\right)\right]+
$$$$
+\frac{\eta_{x y, y}}{G_{x y}}\left(A_{11}^{i} \cos ^{2} \varphi_{i}+A_{21}^{i} \sin ^{2} \varphi_{i}+A_{31}^{i} \sin 2 \varphi_{i}\right)
$$$$
a_{x y}^{(i)}=\frac{1}{G_{x y}}\left[\eta_{x, x y}\left(A_{11}^{i} \cos ^{2} \varphi_{i}+A_{21}^{i} \sin ^{2} \varphi_{i}+A_{31}^{i} \sin 2 \varphi_{i}\right)+\eta_{y, x y}\left(A_{12}^{i} \cos ^{2} \varphi_{i}+A_{22}^{i} \sin ^{2} \varphi_{i}+A_{32}^{i} \sin 2 \varphi_{i}\right)+\right.
$$

$$
\left.+\left(A_{13}^{i} \cos ^{2} \varphi_{i}+A_{23}^{i} \sin ^{2} \varphi_{i}+A_{33}^{i} \sin 2 \varphi_{i}\right)\right]
$$




$$
\begin{aligned}
& b_{x}^{(i)}=\frac{1}{E_{x}}\left[\left(A_{11}^{i} \sin ^{2} \varphi_{i}+A_{21}^{i} \cos ^{2} \varphi_{i}+A_{31}^{i} \sin 2 \varphi_{i}\right)-\mu_{y x}\left(A_{12}^{i} \sin ^{2} \varphi_{i}+A_{22}^{i} \cos ^{2} \varphi_{i}+A_{32}^{i} \sin 2 \varphi_{i}\right)+\right. \\
& -\frac{\eta_{x y, x} E_{x}}{G_{x y}}\left(A_{13}^{i} \sin ^{2} \varphi_{i}+A_{23}^{i} \cos ^{2} \varphi_{i}+A_{33}^{i} \sin 2 \varphi_{i}\right) \\
& b_{y}^{(i)}=\frac{1}{E_{y}}\left[-\mu_{x y}\left(A_{11}^{i} \sin ^{2} \varphi_{i}+A_{21}^{i} \cos ^{2} \varphi_{i}+A_{31}^{i} \sin 2 \varphi_{i}\right)+\left(A_{12}^{i} \sin ^{2} \varphi_{i}+A_{22}^{i} \cos ^{2} \varphi_{i}+A_{32}^{i} \sin 2 \varphi_{i}\right)-\right. \\
& +E_{y} \frac{\eta_{x y, y}}{G_{x y}}\left(A_{13}^{i} \cos ^{2} \varphi_{i}+A_{23}^{i} \sin ^{2} \varphi_{i}+A_{33}^{i} \sin 2 \varphi_{i}\right) \\
& b_{x y}^{(i)}=\frac{1}{G_{x y}}\left[\eta_{x, x y}\left(A_{11}^{i} \sin ^{2} \varphi_{i}+A_{21}^{i} \cos ^{2} \varphi_{i}+A_{31}^{i} \sin 2 \varphi_{i}\right)-\eta_{y, x y}\left(A_{12}^{i} \sin ^{2} \varphi_{i}+A_{22}^{i} \cos ^{2} \varphi_{i}+A_{32}^{i} \sin 2 \varphi_{i}\right)+\right. \\
& \left.+\left(A_{13}^{i} \sin ^{2} \varphi_{i}+A_{23}^{i} \cos ^{2} \varphi_{i}+A_{33}^{i} \sin 2 \varphi_{i}\right)\right) ; \\
& c_{x}^{(i)}=\frac{1}{E_{x}}\left[\left(A_{21}^{i}-A_{11}^{i}\right) \frac{\sin 2 \varphi_{i}}{2}-\mu_{y x}\left(A_{22}^{i}-A_{21}^{i}\right) \frac{\sin 2 \varphi_{i}}{2}+\frac{\eta_{x y, x}}{G_{x y}} A_{33}^{i} \cos 2 \varphi_{i}\right] \text {; } \\
& c_{y}^{(i)}=\frac{1}{E_{y}}\left[-\mu_{y x}\left(A_{21}^{i}-A_{11}^{i}\right) \frac{\sin 2 \varphi_{i}}{2}+\left(A_{22}^{i}-A_{21}^{i}\right) \frac{\sin 2 \varphi_{i}}{2}+\frac{\eta_{x y, y}}{G_{x y}} A_{33}^{i} \cos 2 \varphi_{i}\right] \text {; } \\
& c_{x y}^{(i)}=\frac{1}{G_{x y}}\left[\eta_{x, x y}\left(A_{21}^{i}-A_{11}^{i}\right) \frac{\sin 2 \varphi_{i}}{2}+\eta_{y, x y}\left(A_{22}^{i}-A_{21}^{i}\right) \frac{\sin 2 \varphi_{i}}{2}+A_{33}^{i} \cos 2 \varphi_{i}\right] \text {; } \\
& A_{11}^{(i)}=\bar{E}_{1}^{(i)} \cos ^{4} \varphi_{i}+\bar{E}_{2}^{(i)} \sin ^{4} \varphi_{i}+2\left(\bar{E}_{1}^{(i)} \mu_{12}^{(i)}+2 G_{12}^{(i)}\right) \sin ^{2} \varphi_{i} \cos ^{2} \varphi_{i} ; \\
& A_{12}^{(i)}=A_{21}^{(i)}=\bar{E}_{1}^{(i)} \mu_{12}^{(i)}+\left[\bar{E}_{1}^{(i)}+\bar{E}_{2}^{(i)}-2\left(\bar{E}_{1}^{(i)} \mu_{12}^{(i)}+2 G_{12}^{(i)}\right)\right] \sin ^{2} \varphi_{i} \cos ^{2} \varphi_{i} ; \\
& A_{22}^{(i)}=\bar{E}_{1}^{(i)} \sin ^{4} \varphi_{i}+\bar{E}_{2}^{(i)} \cos ^{4} \varphi_{i}+2\left(\bar{E}_{1}^{(i)} \mu_{12}^{(i)}+2 G_{l 2}^{(i)}\right) \sin ^{2} \varphi_{i} \cos ^{2} \varphi_{i} ; \\
& A_{13}^{(i)}=A_{31}^{(i)}=\left[\left(\bar{E}_{1}^{(i)} \cos ^{2} \varphi_{i}-\bar{E}_{2}^{(i)} \sin ^{2} \varphi_{i}\right)-\left(\bar{E}_{1}^{(i)} \mu_{12}^{(i)}+2 G_{12}^{(i)}\right) \cos 2 \varphi_{i}\right] \sin \varphi_{i} \cos \varphi_{i} \text {; } \\
& A_{23}^{(i)}=A_{32}^{(i)}=\left[\left(\bar{E}_{1}^{(i)} \sin ^{2} \varphi_{i}-\bar{E}_{2}^{(i)} \cos ^{2} \varphi_{i}\right)-\left(\bar{E}_{1}^{(i)} \mu_{12}^{(i)}+2 G_{12}^{(i)}\right) \cos 2 \varphi_{i}\right] \sin \varphi_{i} \cos \varphi_{i} \text {; } \\
& A_{33}^{(i)}=\left[\bar{E}_{1}^{(i)}+\bar{E}_{2}^{(i)}-2 \bar{E}_{1}^{(i)} \mu_{12}^{(i)}\right] \sin ^{2} \varphi_{i} \cos ^{2} \varphi_{i}+G_{12}^{(i)} \cos ^{2} 2 \varphi_{i} .
\end{aligned}
$$




\section{Applied panel design methodology under post-buckling state with combined loading}

Let us consider the case of combined action of longitudinal compressive and tangential flows and rewrite equalities (5) as

$$
\sigma_{x} \delta=-\delta \Omega_{1} f^{2}-q_{x} ; \sigma_{y} \delta=-\delta \Omega_{2} f^{2}-q_{y} ; \tau_{x y} \delta=\delta \Omega_{3} f^{2}-q_{x y} .
$$

Non-linear equation (6) is presented as

$$
f^{2} B_{\alpha \beta} \delta+D_{\alpha \beta} \delta^{3}=\frac{\pi^{2}}{s^{2}} q_{x}+\left(\frac{\pi^{2}}{b^{2}}+\frac{\alpha^{2} \pi^{2}}{s^{2}}\right) q_{y}+\frac{2 \alpha \pi^{2}}{s^{2}} q_{x y} .
$$

where

$$
\begin{gathered}
B_{\alpha s}=\frac{1}{s^{2}}\left[\frac{1}{16 G_{\alpha}}\left(\frac{\alpha}{b}\right)^{2}+\frac{E_{x}}{16} \frac{1}{s^{2}}\right]+\frac{\pi^{2}}{16 G_{\alpha}}\left[\frac{1}{b^{2}}+\frac{\alpha^{2}}{s^{2}}\right]+\frac{\alpha}{s^{2}} \frac{1}{8 b^{2} G_{\alpha}} ; \\
D_{\alpha s s}=\bar{D}_{11} \frac{\pi^{2}}{s^{2}}+2 \bar{D}_{3}\left(\frac{\pi^{2}}{b^{2}}+\frac{\alpha^{2} \pi^{2}}{s^{2}}\right)+\bar{D}_{22}\left[\frac{s^{2}}{\pi^{2}}\left(\frac{\pi^{2}}{b^{2}}+\frac{\alpha^{2} \pi^{2}}{s^{2}}\right)^{2}+\frac{4 \pi^{2} \alpha^{2}}{b^{2}}\right]- \\
-4 \bar{D}_{16} \frac{\alpha \pi^{4}}{s^{4}}-4 \bar{D}_{26} \frac{\alpha \pi^{2}}{s^{2}}\left(3 \frac{\pi^{2}}{b^{2}}+\frac{\alpha^{2} \pi^{2}}{s^{2}}\right) .
\end{gathered}
$$

Let us express the deflection amplitude $f^{2}$ from the nonlinear expression (13) and substitute it into the equations for the total stresses (12), which then are substituted into the expressions for the stresses in the monolayer (10). Then let us put the result of the previous action into the strength criterion (9) and obtain the required nonlinear equation for the panel thickness

$$
\begin{aligned}
& \left(\bar{\sigma}_{2}^{(i)} \tau_{12}^{(i)}\right)^{2}\left[\frac{\left(-\Omega_{1} a_{x}^{(i)}-\Omega_{2} a_{y}^{(i)}-\Omega_{3} a_{x y}^{(i)}\right)}{B_{\alpha s}}\left(\frac{\pi^{2}}{s^{2}} q_{x}+\left(\frac{\pi^{2}}{b^{2}}+\frac{\alpha^{2} \pi^{2}}{s^{2}}\right) q_{y}+\frac{2 \alpha \pi^{2}}{s^{2}} q_{x y}-D_{\alpha s} \delta^{3}\right)+\right. \\
& \left.+q_{x} a_{x}^{(i)}+q_{y} a_{y}^{(i)}+q_{x y} a_{x y}^{(i)}\right]^{2}+ \\
& +\left(\bar{\sigma}_{2}^{(i)} \tau_{12}^{(i)}\right)^{2}\left[\frac{\left(-\Omega_{1} a_{x}^{(i)}-\Omega_{2} a_{y}^{(i)}-\Omega_{3} a_{x y}^{(i)}\right)}{B_{\alpha s}}\left(\frac{\pi^{2}}{s^{2}} q_{x}+\left(\frac{\pi^{2}}{b^{2}}+\frac{\alpha^{2} \pi^{2}}{s^{2}}\right) q_{y}+\frac{2 \alpha \pi^{2}}{s^{2}} q_{x y}-D_{\alpha s} \delta^{3}\right)+\right. \\
& \left.+q_{x} a_{x}^{(i)}+q_{y} a_{y}^{(i)}+q_{x y} a_{x y}^{(i)}\right] \times \\
& \times\left[\frac{\left(-\Omega_{1} b_{x}^{(i)}-\Omega_{2} b_{y}^{(i)}-\Omega_{3} b_{x y}^{(i)}\right)}{B_{\alpha s}}\left(\frac{\pi^{2}}{s^{2}} q_{x}+\left(\frac{\pi^{2}}{b^{2}}+\frac{\alpha^{2} \pi^{2}}{s^{2}}\right) q_{y}+\frac{2 \alpha \pi^{2}}{s^{2}} q_{x y}-D_{\alpha s} \delta^{3}\right)+\right. \\
& \left.+q_{x} b_{x}^{(i)}+q_{y} b_{y}^{(i)}+q_{x y} b_{x y}^{(i)}\right]+ \\
& +\left(\bar{\sigma}_{l}^{(i)} \tau_{12}^{(i)}\right)^{2}\left[\frac{\left(-\Omega_{1} b_{x}^{(i)}-\Omega_{2} b_{y}^{(i)}-\Omega_{3} b_{x y}^{(i)}\right)}{B_{\alpha s}}\left(\frac{\pi^{2}}{s^{2}} q_{x}+\left(\frac{\pi^{2}}{b^{2}}+\frac{\alpha^{2} \pi^{2}}{s^{2}}\right) q_{y}+\frac{2 \alpha \pi^{2}}{s^{2}} q_{x y}-D_{\alpha s} \delta^{3}\right)+\right. \\
& \left.+q_{x} b_{x}^{(i)}+q_{y} b_{y}^{(i)}+q_{x y} b_{x y}^{(i)}\right]^{2}+ \\
& +\left(\bar{\sigma}_{1}^{(i)} \bar{\sigma}_{2}^{(i)}\right)^{2}\left[\frac{\left(-\Omega_{1} c_{x}^{(i)}-\Omega_{2} c_{y}^{(i)}-\Omega_{3} c_{x y}^{(i)}\right)}{B_{\alpha s}}\left(\frac{\pi^{2}}{s^{2}} q_{x}+\left(\frac{\pi^{2}}{b^{2}}+\frac{\alpha^{2} \pi^{2}}{s^{2}}\right) q_{y}+\frac{2 \alpha \pi^{2}}{s^{2}} q_{x y}-D_{\alpha s} \delta^{3}\right)+\right.
\end{aligned}
$$




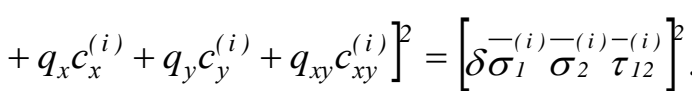

It is known that, as a rule, standard reinforcement angles of $0^{\circ}, \pm 45^{\circ}, 90^{\circ}$ are used for elements of aircraft structures. For anisotropic panels, stacks with different relative thicknesses are used to achieve the effect of anisotropy. In this case, it is necessary to consider four equations of the form (14) to indicated reinforcement angles and then select the maximum value from the resulting numerical thickness solutions.

Also, it is necessary to note that in the combined case of loading the critical parameters of wave formation are determined numerically from relations (8) from the linear equation (7). Further, in the general case, it is also necessary to select the potential critical points numerically, where stresses (5) reach their maximum values. In some cases, taking into account the form of expressions for the quantities $\Omega_{1}, \Omega_{2}$ and $\Omega_{3}$, in equalities (5), the coordinates of the potentially critical points of the panel can be found from the condition

$$
\cos 2 \pi\left(x-\alpha_{\text {крum }} y\right) / s_{\text {крum }} \rightarrow 1 .
$$

\section{Discussion of the design methodology of rectangular composite panel for the post-buckling state under longitudinal compression and shear}

Relation (14) is the most general case in terms of loading and taking into account the peculiarities of the composite structure. Let us consider, for example, the case of a composite panel with given relative thicknesses with reinforcement angles of $0 \%+45^{\circ}$ under the effects of longitudinal compressive and tangential flows.

As a rule, at the early stages of structure's design without taking into account the safe-life time requirements under the conditions of static strength, a criterion is used in accordance with the 1st theory of strength $\sigma_{I}^{(i)}=\bar{\sigma}_{I}{ }^{(i)}$ for composite materials. Then the general expression (14) can be rewritten as

$$
\left[\frac{\left(-\Omega_{1} a_{x}^{(i)}-\Omega_{2} a_{y}^{(i)}-\Omega_{3} a_{x y}^{(i)}\right)}{B_{\alpha s}}\left(\frac{\pi^{2}}{s^{2}} q_{x}+\frac{2 \alpha \pi^{2}}{s^{2}} q_{x y}-D_{\alpha s} \delta^{3}\right)+q_{x} a_{x}^{(i)}+q_{x y} a_{x y}^{(i)}\right]^{2}=\left[\delta \bar{\sigma}_{l}^{(i)}\right]^{2}
$$

Where the factors from equations (11)

$$
\begin{gathered}
a_{x}^{(0)}=\bar{E}_{1}^{(0)} \frac{1}{E_{x}}\left(1-\mu_{12}^{(0)} \mu_{x y}\right), a_{y}^{(0)}=\bar{E}_{1}^{(0)} \frac{1}{E_{y}}\left(\mu_{12}^{(0)}-\mu_{y x}\right), a_{x y}^{(0)}=\frac{\bar{E}_{1}^{(0)}}{G_{x y}}\left[\eta_{x, x y}+\eta_{y, x y} \mu_{12}^{(0)}\right] ; \\
a_{x}^{(45)}=\bar{E}_{1}^{(45)}\left[\frac{1}{2 E_{x}}\left(1-\mu_{y x}\right)\left(1+\mu_{12}^{(45)}\right)+\frac{\eta_{x y, x}\left(1-\mu_{12}^{(45)}\right)}{2 G_{x y}}\right] ; \\
a_{y}^{(45)}=\bar{E}_{1}^{(45)}\left[\frac{1}{2 E_{y}}\left(1-\mu_{x y}\right)\left(1+\mu_{12}^{(45)}\right)+\frac{\eta_{x y, y}\left(1-\mu_{12}^{(45)}\right)}{2 G_{x y}}\right] ; \\
a_{x y}^{(45)}=\frac{\bar{E}_{1}^{(45)}}{G_{x y}}\left[\frac{\eta_{x, x y}\left(1+\mu_{12}^{(45)}\right)}{2}+\frac{\eta_{y, x y}\left(1+\mu_{12}^{(45)}\right)}{2}+\frac{\left(1-\mu_{12}^{(45)}\right)}{2}\right]
\end{gathered}
$$

In this case, the design should be carried out taking into account the effects of the calculated loads with a safety factor $f=1.5$.

When the structure design is taking into account the safe-life time requirements, it is possible to use the condition of non-cracking of the binder and the criterion of strength (9) as

$$
\left(\frac{\sigma_{2}^{(i)}}{\bar{\sigma}_{2}^{(i)}}\right)^{2}+\left(\frac{\tau_{12}^{(i)}}{\bar{\tau}_{12}^{(i)}}\right)^{2}=1
$$


Then the formula (14) is reduced also:

$$
\begin{gathered}
\left(\bar{\tau}_{12}^{(i)}\right)^{2}\left[\frac{\left(-\Omega_{1} b_{x}^{(i)}-\Omega_{2} b_{y}^{(i)}-\Omega_{3} b_{x y}^{(i)}\right)}{B_{\alpha s}}\left(\frac{\pi^{2}}{s^{2}} q_{x}+\frac{2 \alpha \pi^{2}}{s^{2}} q_{x y}-D_{\alpha s} \delta^{3}\right)+q_{x} b_{x}^{(i)}+q_{y} b_{y}^{(i)}+q_{x y} b_{x y}^{(i)}\right]^{2}+ \\
+\left(\bar{\sigma}_{2}^{(i)}\right)^{2}\left[\frac{\left(-\Omega_{1} c_{x}^{(i)}-\Omega_{2} c_{y}^{(i)}-\Omega_{3} c_{x y}^{(i)}\right)}{B_{\alpha s}}\left(\frac{\pi^{2}}{s^{2}} q_{x}+\frac{2 \alpha \pi^{2}}{s^{2}} q_{x y}-D_{\alpha s} \delta^{3}\right)+q_{x} c_{x}^{(i)}+q_{y} c_{y}^{(i)}+q_{x y} c_{x y}^{(i)}\right]^{2}=\left[\delta \bar{\sigma}_{2}^{(i)} \bar{\tau}_{12}^{(i)}\right]^{2} .
\end{gathered}
$$

In this case, the design must be carried out under the effects of service loads with a safety factor $f=1.0$. Note that the coefficients $a_{m}{ }^{(i)}, b_{m}{ }^{(i)}$ and $c_{m}{ }^{(i)}$ from equalities (11) can be transformed to the form

$$
\begin{gathered}
b_{x}^{(0)}=\bar{E}_{2}^{(i)}\left[\frac{\mu_{21}^{(0)}}{E_{x}}-\frac{\mu_{y x}}{E_{y}}\right] ; b_{y}^{(0)}=\bar{E}_{2}^{(0)}\left[\frac{1}{E_{y}}-\frac{\mu_{x y} \mu_{12}^{(0)}}{E_{x}}\right] ; b_{x y}^{(0)}=\frac{1}{G_{x y}}\left[\eta_{x, x y} \mu_{21}^{(0)}+\eta_{y, x y}\right] ; \\
c_{x}^{(0)}=G_{I 2}^{(0)} \frac{\eta_{x y, x}}{G_{x y}} ; c_{y}^{(0)}=G_{I 2}^{(0)} \frac{\eta_{x y, y}}{G_{x y}} ; c_{x y}^{(0)}=\frac{1}{G_{x y}} G_{12}^{(0)} ; \\
b_{x}^{(45)}=\bar{E}_{2}^{(45)}\left[\frac{1+\mu_{21}^{(45)}}{2 E_{x}}-\frac{\mu_{y x}}{2 E_{y}}\left(1+\mu_{12}^{(45)}\right)-\frac{\eta_{x y, x}\left(1-\mu_{12}^{(45)}\right)}{2 G_{x y}}\right] ; \\
b_{y}^{(45)}=\bar{E}_{2}^{(45)}\left[\frac{1+\mu_{21}^{(45)}}{2 E_{y}}-\frac{\mu_{x y}}{2 E_{x}}\left(1+\mu_{12}^{(45)}\right)-\frac{\eta_{x y, y}\left(1-\mu_{12}^{(45)}\right)}{2 G_{x y}}\right] ; \\
b_{x y}^{(45)}=\frac{1}{G_{x y}}\left[\frac{\eta_{x, x y}\left(1+\mu_{21}^{(45)}\right)}{2}+\frac{\eta_{y, x y}\left(1+\mu_{12}^{(45)}\right)}{2}-\frac{\left(1-\mu_{12}^{(45)}\right)}{2}\right] ; \\
c_{x}^{(45)}=G_{I 2}^{(45)}\left(\frac{1+\mu_{x y}}{E_{x}}\right) ; c_{y}^{(45)}=G_{I 2}^{(45)}\left(\frac{1+\mu_{x y}}{E_{y}}\right) ; c_{x y}^{(45)}=G_{12}^{(45)}\left(\frac{\eta_{y, x y}+\eta_{x, x y}}{G_{x y}}\right) .
\end{gathered}
$$

Conditions (15) allow to simply the expressions (16)-(17) also.

\section{Conclusions}

1. The paper proposes an applied technique for determining the minimum thickness of an anisotropic panel for supercritical behaviour under complex combined loading. The technique is based on the analytical solution of a geometrically nonlinear problem.

2. For a multilayer composite package consisting of $n$ layers, the design problem is generally reduced to a system of nonlinear equations relative to the desired panel thickness. With it is necessary to satisfy the Tsai's criterion, which is important when a structure's design is taking into account the requirements of not only static strength but safe-life time also.

3. This paper presents the design technique for the post-buckling state in the most general feature when the strength criterion for the ultimate combination of stresses is achieved in the monolayer. The practical significance of the work is the possibility of using the obtained relations in any mathematical package such as Mathcad and the subsequent expert determination of panel thicknesses, taking into account the geometrically nonlinear behaviour under combined loading at the early design stages.

\section{References}

[1] Advisory Circular 20-107B, U.S. Department of Transportation Federal Aviation Administration, 2009.

[2] Barkanov E., Gluhih S, Ozolins Olgerts, Eglítis E., Almeida F., Bowering M., Watson G. (2010). Optimal weight design of laminated composite panels with different stiffeners under buckling loads. 27th Congress of the International council of the Aeronautical sciences 2010, ICAS 2010. 3. pp. 2030-2038. 
[3] Lim W.-K., Jeong W.-K., Tschegg E. Failure of fibrous anisotropic materials under combined loading. Composites. Part B: Engineering. 41. 2010, pp. 94-97.

DOI: 10.1016/j.compositesb.2009.06.001.

[4] Falzon B. G., Aliabadi M. (2008). Buckling and Postbuckling Structures: Experimental, Analytical and Numerical Studies. DOI: 10.1142/p506.

[5] Faggiani A., Falzon B.G. Optimization of Postbuckling-Stiffened Composite Aerostructures: Experimental, Analytical and Numerical Studies, 2018, DOI: 10.1142/9781786344335_0008.

[6] Vasiliev V. V., Morozov E. V. Advanced Mechanics of Composite Materials and Structures. Amsterdam: Elsevier, 2018, 900 p.

[7] Nemoto K., Kikugawa H., Moriyama H., Kasuya H. Effect of lamination constitution on postbuckling behavior of symmetrically laminated plates with initial deflections. 57, 2016, pp. 654-660. DOI: 10.2320/matertrans.M2016015.

[8] Nemoto K., Kikugawa H., Moriyama H., Kasuya H. Effect of Lamination Constitution on PostBuckling Behavior of Symmetrically Laminated Plates with Initial Deflection. Journal of the Japan Institute of Metals. 79. 2015, pp. 49-56. DOI: 10.2320/jinstmet.J2014041.

[9] Mitrofanov O. Applied design of minimum weight composite wing panels taking into account skin post-buckling behavior // MAI review. Vol. 9. 2002. No.1, pp. 34-41.

[10] Mitrofanov O. Post-Buckling State Estimation and Load-Bearing Composite Panels Design for Compression and Shear. AIP Conference Proceedings 2125, 030059 (2019); DOI: $10.1063 / 1.5117441$.

[11] Sirotkin O.S., Grishin V.I., Litvinov V.B. Design, calculation and technology of aircraft structures joints (Moscow: Mechanical engineering), 2006, 330 p. 